\title{
On the Performance Analysis of Recursive Data Replication Scheme for File Sharing in Mobile Peer-to-Peer Devices Using the HyMIS Scheme
}

\author{
Constandinos X. Mavromoustakis and Helen D. Karatza \\ Department of Informatics \\ Aristotle University of Thessaloniki \\ 54124 Thessaloniki, Greece \\ \{cmavrom,karatza\}@csd.auth.gr
}

\begin{abstract}
Advances in wireless networks enable high rates interaction between mobile devices. Short-range wireless communication technologies such as wearable PCs demand low latency and reliability as the first thing for considering QoS. Mobile Peer-to-Peer devices as an autonomous system of mobile routers that are selforganized, self-configured and completely decentralized are characterized by bounded resource sharing reliability. Due to the uncertainty in available resources wireless networks could rarely host file sharing applications in a reliable manner. This paper examines the response of a gossip-based data replication scheme for reliable file sharing under specified patterns and conditions, using the Hybrid Mobile Infostation System (HyMIS). This scheme is based on the advantages of mobile Infostations. Combining the strengths of autonomic gossiping and the hybrid -entirely mobileInfostation concept, this scheme enables end to end reliability. Examination is performed for the response, the robustness and the offered reliability while examining the effectiveness of the proposed scheme for facing mobility limitations using the gossip-based 'selection' of users.
\end{abstract}

\section{Introduction}

In modern applications the concept of mobility comes in many forms and patterns. Recent infrastructure based networks can support online gaming and multimedia services in a supported full mobility devices. Different network configurations like Mobile Ad hoc Networks (MANets), Mobile peer to peer systems (MP2P) or hybrid systems (Infostations [1]) take advantages of user's mobility to improve connectivity, information sharing and sensor characteristics. Today the ubiquitous presence of mobile phones and PDAs equipped with close range radio connectivity like Bluetooth and WLAN create a fertile environment for ad hoc network applications. Many applications and platforms are being born with the new wireless technology formation like file sharing platforms, directed advertisement for mobile commerce and tourism, or dissemination of metadata about services being provided.

Choosing appropriate information dissemination strategies is crucial in MANets due to the frequent and unexpected changes in topology. Applying epidemiological models to information diffusion allows the evaluation of such strategies depending on the MANET characteristics, e.g. the node density. In order to choose appropriate strategies at run time, the model should be easily evaluated. Reliability in highly changing topology networks is a major issue since their moving characteristics trade off the end user's QoS.

In an infrastructure based wireless systems hosts are purely connected and "long lived". However in wireless systems where no infrastructure exists like MP2P systems, hosts/devices are "short-lived". Connections between peers on the network are prone to failures while this is aggravated by short connection times, unpredictable disconnections (range and battery failure), small network formation factor, and file's unavailability. This paper proposes a reliable file sharing scheme for MP2P devices merging the advantages of epidemic file dissemination through purely mobile Infostations (on the contrary with static presented in $[1,9,10])$ using the proposed Hybrid Mobile Infostation System (HyMIS). All mobile users are structured in the landscape in a way that is based on their location at certain time. Each mobile user can become a Primary Infostation after being a candidate (cPI) for which some criteria need to be matched. This scheme proved its scalability and does not require spatial distributions for efficient spread of information in supported mobility. The paper is organized as follows: Section 2 discusses the related work that has been done for MP2P reliable information sharing, featuring out the basic principles and the conducted solutions by different schemes. Section 3 then introduces 
the proposed reliable file sharing scheme for MP2P users using the proposed HyMIS structure and the different approach in supported mobility. Section 4 provides the evaluation and simulation results of the proposed scheme in contrast to the reliability factors for file sharing in MP2P hybrid networks followed by section 5 which concludes with a summary of our contribution and further research.

\section{Related work}

The information sharing process in a dynamically changing topology environment requires a minimal amount of time for being efficient. This interval is very crucial for the QoS provided. In dense networks, the contention and collisions caused by the random interactions of neighboring nodes harms the performance since file sharing techniques rely on overhearing. This issue can be disastrous for sensitive information since time and capacity limitations can take place at each node due to overhearing, and needed information will be lost.

When diffusing data in an infrastructureless wireless network, naive retransmission of broadcasts can lead to the broadcast storm problem [2] where redundancy, contention, and collisions impair performance and reliability. Flooding in a wireless network is in fact as efficient as wired networks because of wireless multicast advantage. In [3] authors identify several interesting effects at the link-layer with more noticeable the highly irregular packet reception contours, the likeliness of asymmetric links, and the complex propagation dynamics of simple protocols. In [4] an epidemic algorithm is proposed based on strictly local interactions for managing replicated databases in a robust way for unpredictable communication failures. The epidemic property is important since MP2P devices experience high loss rates due to their unpredictable movements, their asymmetric connectivity, and to node failures in the "repopulation" process [5]. When a mobile node makes an explicit request for a resource, the whole network is flooded with a query, like mobile ad-hoc route discovery algorithms [6]. Data replication schemes are proposed in [7] for ad hoc networks which are based on the improvement of data accessibility. However, this approach may not be valid when the link failure probability is taken into consideration. Another drawback is that it only considers the accessibility, without considering the query delay. Significant improvements of the basic flooding approaches using advertisements and geographic information have also been recently studied [8]. This work proposes a novel approach for reliable landcast (efficient interlandcast resource sharing) using the HyMIS configuration. This scheme implements a variant of mobile Infostation concept proposed in [22]. In [22] a partially centralized supervision takes place in order to enable efficient Geocast dissemination. On the contrary this work uses the purely mobile Infostation concept where different users could become candidates for being a Primary Infostation (cPI) and being the carriers for delivering packets to other users. One basic advantage is that this scheme does not flood the whole network with any other control transmissions. It only "builds" a tree of PIs and MIs as presented in the following section. HyMIS architecture could be the basis for reliable file sharing among hybrid platforms.

\section{Epidemic replication for maintaining file sharing reliability using the Hybrid Mobile Infostation System (HyMIS)}

This section describes the reliable autonomous file sharing scheme with use of the purely mobile Infostations, which bounds the dissemination of requested files in certain geographical landscapes (flexible Geocasting) in an epidemic way.

\subsection{Purely mobile Infostations in organized landscapes}

Systems based on "run anywhere, anytime, anything" concept in pervasive environments, demands ensured QoS. These systems are based on the waterfilling concept, where the transmission occurs only when source and destination are close together to ensure reliability. Results obtained for these issues [1, 9, 11] have provided good estimates that permit such systems to become the basis for low cost, wireless data transmission.

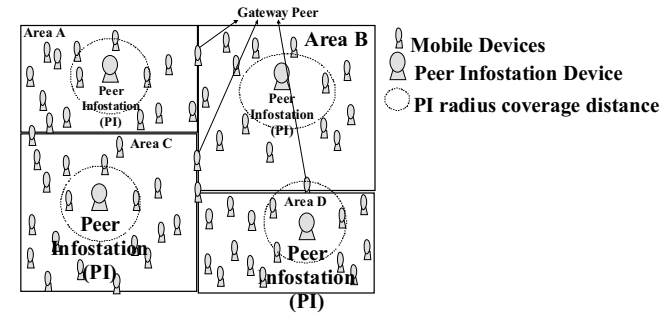

Figure 1: Four landscapes with the purely mobile Infostation concept applied in each landscape.

Wireless networks due to device limitations have constrained capacities and bounded capabilities in terms of reliable networking. Some research results presented in [10], introduce mobility into a fixed network model, which show that the average throughput per sourcedestination pair can be kept constant even if the number of nodes per unit area increases. This improvement is obtained through the exploitation of the time-variation of the user's channel due to mobility. The new concept in [10] is to split the packets of each source node to as many nodes as possible. Therefore, strategies of this type incur additional delay, possible jitter in the reconstruction process because packets have to be buffered until the 
channel becomes sufficiently strong for transmission(s). Taking into account all the above reasons, this paper adopts the idea of a hybridized version of static Infostation concept [22] and propose a layered scheme using a non-static Infostation but a purely mobile. Figure 1 shows the basic mobile Infostation concept in a fourregional geographic landscape. The Primary Infostation (PI) is mainly temporary and acts as a carrier to other users in the same landscape. If energy, capacity or velocity limitations arise, then PI subsequently enables other peer user that matches the PI chosen criteria (a candidate PI (cPI)), to become a PI.

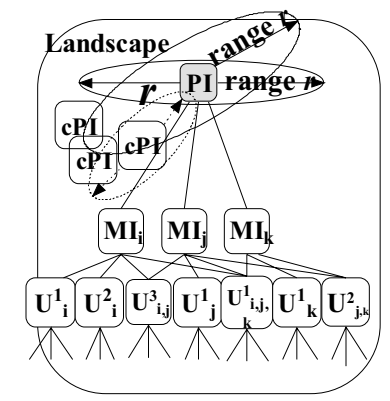

Figure 2: The hybrid peer-to-peer mobile Infostation based architecture.

Selected devices that play the role of broadband hotspots (like PI) in a centralized form is a way to improve reliability. This concept is already implemented through Infostations [1]. Different locations and characteristics of user mobility patterns for different scenarios are depicted in $[11,12]$. This work combines the pure Infostation system with a mobility based framework introducing the Hybrid Mobile Infostation System (HyMIS) where the primary Infostation is not static (PI) but can move with lower velocity ${ }^{\prime}$ than other selected Infostations. Secondary mobile Infostations could move across an area forming a cluster with other peers and exchanging information with PI of each landscape. PI is not only selected if it is set in the centre of the landscape/geographic area as proposed in [22] but any wireless device could become a $\mathrm{PI}^{2}$ if the residual energy is high enough (higher than other cPIs), the frequency of changing landscape is low and remaining capacity is high enough. A cPI becomes a PI if it matches all the criteria or the best "matched" user. PI covers only a specified circle of radius $R$. The $R$ radius coefficient depends (as in all mobile hosts) to signal power.

The PI capacity node plays a role of control storage node [13]. On the contrary to [13] this work proposes a hybrid peer-to-peer tree based structure shown figure 2 .

\footnotetext{
That might be research criterion for selecting a device to be PI.

Matching the described criteria.
}

PI is selected ${ }^{3}$ and located in the center of every landscape covering a certain predetermined area. A cPI mobile peer is chosen to be PI only if it has high residual energy and capacity and moves with low velocity (or stall). This means that for a certain time distance $\mathrm{T}$ (until it remains as the PI of the landscape) user $U_{q(i)}^{1}$ will match all selection criteria until other cPI will substitute current PI. This principle is shown in figure 2. As depicted in figure 2, $U_{i, j, k}^{1} \quad$ is downloading simultaneously from $M I_{i}, M I_{j}, M I_{k}$ or vice versa. In the coverage of PI some cPIs are approaching to become a PI based on the criteria previously explained. If current PI does not match the set criteria any more, then the best matching $\mathrm{cPI}$ becomes a PI. This mechanism occurs recursively, combined with the epidemic model that is explained in the following section. The coexistence of PIs (that do not change landscape every while) and MIs, simultaneously recovers from the split of the peer-to-peer network, and improves the network topology connectivity. In order to improve the inefficiency of the pure peer-to-peer flooding tactic an epidemic object replication scheme is chosen for reliable file sharing, which is described in the following section.

\subsection{Epidemic object replication scheme for reliable file sharing}

Directed dissemination scenarios, or epidemic algorithms, which follow a nature paradigm, enable local view of the environment. According to this fact, epidemic algorithms are easy to implement and guarantee message propagation in heterogeneous environments. Reliability in file sharing can be determined by relying on epidemic algorithms, a breed of distributed algorithms that find inspiration in the theory of epidemics. It enables a virtual motoring of a group of devices sharing common resource(s) (or directed "infections" see figure 2). Based on certain characteristics, epidemic algorithms are amenable to the highly dynamic scenarios. This work uses a promiscuous caching scenario which means that data can be cached "anywhere, anytime". However users due to their mobility reduce the reliability, causing sudden network partition or network split. Thus a proactive dissemination scheme must be determined in order to prevent the cutoff in file sharing communication. This work assumes an isolated system comprising of a fixed number of mobile nodes confined in a predefined geographic region (landscape). These nodes are mobile, and communicate with each other in a wireless (radio) adhoc manner. As studied in $[8,10,12]$ there is a trade off

\footnotetext{
Selection is based on the low velocity criterion and trajectory of each device
} 
between reliable coverage and data rate. The limited connectivity coverage that MP2P systems offer, results in significant delay in downloading a message or file (group of packets). In the proposed scenario each node carries some unique data items.

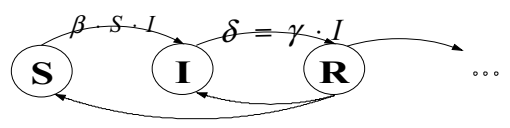

Figure 3: Markov chain model of an infectious disease with susceptible, infected, and recovered states.

Each mobile host $m_{k}$ has a predetermined capacity $M$. At any time in the network each $m_{k}$ has a state. There are three states that $m_{k}$ can be characterized: the susceptible state $S(t)$ represents the number of hosts in the system which are "susceptible," infected state $I(t)$ represents the number of "infected" hosts, and $R(t)$ represents the "recovered" hosts. A host is in susceptible state $S(t)$ if the device does not share any information with any other host. In turn A host is in infected state $I(t)$ if a file(s) share occurs. Finally a host is in "recovered" state $R(t)$ if any shared file(s) are no longer pending. A Markov chain model of an infectious disease with susceptible, infected, and recovered states is used shown in figure 3. Markov chain model was chosen to evaluate this extra storage requirement and to determine the file sharing termination criteria.

The modelling of diseases in fixed networks have been studied in the past [14], and this model is used in a similar manner in this scenario. Adopting the framework from an infectious disease model [15], a host is set as "infected" if a file sharing (or a group of data packets) are pending. Suppose there are $k$ hosts in the system, then a host is sharing a resource with $\beta(k-1)$ other hosts per unit time. $S$ $(k-1)$ do not have yet the disease. Therefore, and according to [22] the transition rate from state $\mathrm{S}$ to state I becomes

$$
\text { Filesharing }=I[\beta(k-1)] \cdot\left[\frac{S}{k-1}\right]
$$

where $\beta$ is the contact rate for $k$ hosts.

Then the downloaded (no longer pending) rate is:

$$
\delta=\gamma \cdot I
$$

where $\gamma$ is the download rate and $I$ is the number of infected devices.

The $\beta \cdot S \cdot I$ is called $\pi$ coefficient which indicates the enforcement degree of the diffusion process. $\pi$ has the dimension of $\left[\frac{1}{\text { Time }}\right]$. Previous examinations of the behaviour of small scale systems [15] showed that relatively small populations could be faced wit a stochastic model. Thus taking into account that $\pi$ depends on the number of $S(t)$ and $I(t)$ and the probability of transmitting the information, we can derive $S(t)$, solve the first order differential equation [as in 22] and evaluate the cumulative distribution function (3).

$$
\begin{aligned}
& I^{\prime}(t)=\frac{I(t)}{N}=\frac{N}{N \cdot\left(1+e^{-\beta \cdot N \cdot t}(N-1)\right)} \\
& I^{\prime}(t)=\frac{1}{1+e^{-\beta \cdot N \cdot t}(N-1)}
\end{aligned}
$$

The average delay experienced by all the peers in downloading a multi-part file is as follows:

$$
\bar{d}^{(m)} \approx \frac{\tau_{0}}{m} \log _{2} n
$$

where $m$ is the number of identical sized chunks that the file is divided, $n$ is the number of peers and $\tau_{0}$ is the amount of time taken to download the whole file, if downloaded from a single peer.

By using the above analytical epidemic approach for packet diffusion the gain is significant as results depict in the following section. Epidemic approach with the highly redundant and fault tolerant way is applied in the system only when a packet is disturbed on its way to destination or when sender or receiver notice an unreliable communication. This might be an unpredictable movement, asymmetric connectivity and node failures. There exist two different user-based cases where the communication might be disturbed: (i) when source user's communication fails and (ii) user's destination communication fails. In case (i) the source users might move to a point that no communication coverage exists and as a result connection failure will occur and the prospective resource for download will be lost.

Taking cases (i) and (ii) as a paradigm this paper proposes a solution in order to enable reliability in resource sharing for MP2P users. Considering a file ${ }^{4}$ download from a node A to a node B it is helpful to evaluate both cases. In (i) the user's device (mobile node) chooses in epidemic form (infection) which of user's device neighboring nodes, will be MI. This classification is based on candidate's node residual energy, capacity and signal transmission power [21]. PI only communicates with MI and not with pure users. Node "A" then copies packets to the chosen "infected" MI for time $t, t>\varphi_{\mathrm{T}}$, where $\varphi_{\mathrm{T}}$ is the time estimated for complete download. MI in turn copies these packets to PI of the landscape to which is the only user which communicates directly [13, 21]. This file lies in PI buffer for time $t$ and then is deleted. This mechanism occurs recursively for the forthcoming chosen MIs, in the case where MI's communication fails. Finally when downloading is completed, file is being removed from PI and "infected" MI even if time $t, t>\psi_{\mathrm{T}}$, has not yet ended. Then every infected node recovers. If any change in PI occurs, then PI

\footnotetext{
Stream(s) of packets.
} 
copies all requested packets to the new PI and to (one) best chosen $\mathrm{cPI}$.

In case (ii) where the destination node " $\mathrm{B}$ " is to be moved influencing the communication between $\mathrm{A}$ and $\mathrm{B}$, a similar mechanism is activated in the following way: if the signal transmission power is reduced which means communication between A-B is prone to failure(s), " $\mathrm{B}$ " sends messages to "A's" neighbors to search for MIs in an epidemic way-as explained earlier. In turn MIs copy packets to PI of the landscape and then PI to MI-cluster controllers to which an examination takes place to examine whether destination node " $\mathrm{B}$ " changed landscape. If " $\mathrm{B}$ " changed landscape then MI-cluster controllers copy file packets to selected users in the nearby landscape and those users are responsible to designate a copy to PI of the landscape otherwise the epidemic algorithm of case (i) takes place. The above mechanism occurs recursively until peers complete their download(s).

Epidemic scheme supports the eventual delivery of messages to arbitrary destinations with minimal assumptions regarding the underlying topology and connectivity of the underlying network. Assuming that the mobility of hosts is a hybridized version of city walk and random walk [16] the epidemic selective method is applied only when any file transfer is disturbed. Each host chooses a target node randomly as its next destination, and moves toward this target at a variable speed parameterized by average speed. Epidemic packet diffusion enables reliability as shown in the results in the following section.

\section{Simulation experiments and discussion}

We assume a system consisting of several mobile nodes, e.g., mobile users equipped with notebooks or PDAs and wireless network interfaces. All mobile nodes collaborate via a shared application that uses a distributed lookup service. Radio coverage is small compared to the area covered by all nodes, so that most nodes cannot contact each other directly. The underlying radio technology is the well known IEEE 802.11x. However, it is necessary to point out that communication and epidemic-like dissemination could be employed on any radio technology that enables broadcast transmissions inside a node's radio coverage.

\subsection{Routing protocol used}

In the implementation of the proposed scenario the Zone Routing Protocol (ZRP) $[19,20]$ is used. The ZRP is considered advantageous because allows to a certain node to accurately know the neighbors of any mobile terminal within a zone (PI-like approach [1, 2, 6, 19]). In the proposed scenario zone refers to each node's connectivity domain as appears in the tree of figure 2. ZRP allows the routing protocol to be adjustable for different operational network conditions such as heavy traffic [17-21].

\subsection{Simulation and performance evaluation of the HyMIS scenario}

In this section, some experimental and simulation results are presented for the performance evaluation and reliability in resource sharing, offered by the proposed scheme. We evaluated the epidemic caching using the HyMIS configuration and the grade of HyMIS structure contribution for enabling reliability in file sharing. Network partitioning that degrades system's performance and latency issues that arise, are also been examined.

In simulation a two-dimensional network was used, 4 landscapes each one consisting of 25 nodes with each link (frequency channel) having max speed reaching $2 \mathrm{Mb}$ per sec. The propagation path loss is the two-ray model without fading. The network traffic is modeled by generating constant bit rate (CBR) flows. Each source node transmits one 512-bytes ( $\sim$ Kbits-light traffic) packet. Packets generated at every time step by following Pareto distribution as depicted in [17, 18], destined for a random destination uniformly selected. Network structure has been implemented as a [N-1] row, [N-1] column for each node being a possible destination as developed in [21]. Two sets of experiments were performed: examination for the accuracy of the selective replication concept and the grade of contribution in enabling reliability in file sharing. The second deals with the performance under significant traffic, network partition limitations and the latency issues that arise. Caching capacity of each node could be unlimited while nowadays memory becomes cheaper and cheaper. However if each node has unlimited capacity then enormous traffic will reach each node's buffer causing "vicious" dissemination in a bounded node network. Thus taking into account this issue, this paper evaluates two different types of replication/caching capacity:

(i). Unlimited capacity

(ii). Limited capacity for each node as $64 \mathrm{~KB}, 128 \mathrm{~KB}$, $512 \mathrm{~KB}, 2 \mathrm{MB}, 10 \mathrm{MB}$.

An issue that has to be taken into account is whether the cached information destined for a proper node could be stored in a node with higher residual energy. As shown in simulation process if nodes with higher level of residual energy are chosen in the path then the network partitioning probability is further reduced [21]. For this reason cached information size and file size are chosen randomly in our scenario and files are searched for, upon queries on a recursive basis. 


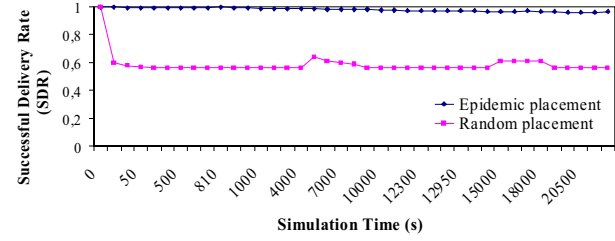

Figure 5: The ratio of successful packet delivery.

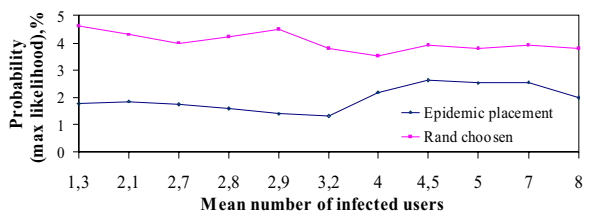

Figure 6: Mean number of infected users in the landscape.

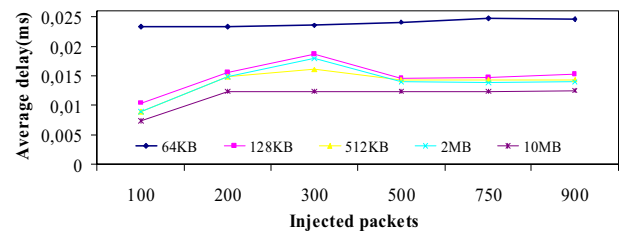

Figure 7: Average packet delay versus the number of injected packets in the network.

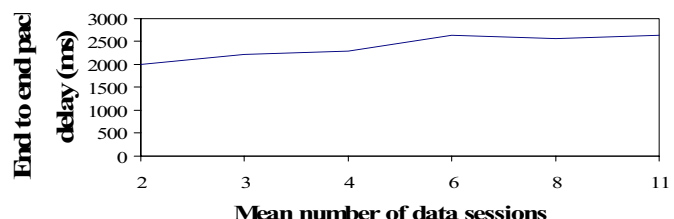

Figure 8: Mean number of End to end packet delay (ms) with the mean number of data sessions for a complete download.

Figure 5 shows the successful packet delivery ratio (SDR). The SDR metric is the number of certain number of packets sent over the total number of packets sent. From figure 5 it is shown that the SDR drops slightly using the epidemic selection. Compared with randomly selected users (even for cache placement or) the epidemic diffusion behaves significantly better. After consecutive simulations the extracted values did not dropped below $98.5 \%$ for successful delivery of packet using the HyMIS structure.

Figure 6 illustrates the mean number of infected users, infected by epidemic algorithm used and by randomly chosen selection, in the landscape. It can clearly be considered that the mean number of infected users for which epidemic algorithm is used is significantly small compared with the number of randomly chosen. This proves the robust characteristics in node selection for packet's caching ("matched" criteria) in order to enable reliable file sharing. Also it is shown that the Markov model chain for completing a download, bias in an epidemic form the other users. This means that more users get infected that with the random selection which is illustrated and experimentally in figure 6 .

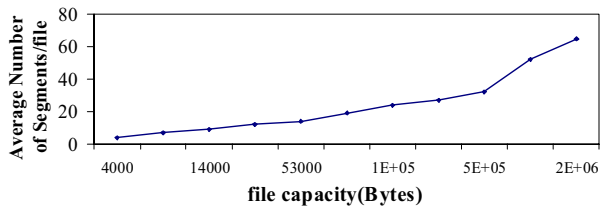

Figure 9: Average number of segments/file versus file capacity

Figure 7 illustrates the average packet delay with the number of injected packets in the network. As the number of packets increases the average packet delay slightly increases. After consecutive simulations for this metric it has been shown that when the number of injected packets reaches 250, the average packet delay increases dramatically for $128 \mathrm{~KB}, 512 \mathrm{~KB}, 2 \mathrm{MB}$ and $10 \mathrm{MB}$ capacities. This occurs due to capacity limitation that binds each node. For different capacity limitations the average delay remains almost the same except the case of $64 \mathrm{~KB}$ for which the average delay increases due to the bounded capacity.

Figure 8 shows mean number of the end to end packet delay $(m s)$ with the mean number of data sessions for a complete download. Some downloads could be corrupted during packet transfers. Figure 8 shows the measures for the session's count ${ }^{5}$ with the corresponding end to end packet delay. It is shown that for high session counts the end to end packet delay seems to be in terms of sec which is dramatic for multimedia transmissions. For a minimal of 2 sessions the end to end packet delay is almost $2 \mathrm{sec}$.

In figure 9 the average number of segments per file versus the capacity of each file is illustrated. For large files $(>1 \mathrm{MB})$, the segments which are randomly chosen depending on each file chunks, are not exceeding 28 . In figure 9 it is indicated that small files can be segmented (in ratio) in much more pieces than for large files. This results a better reliability response in many consecutive simulation's potshots.

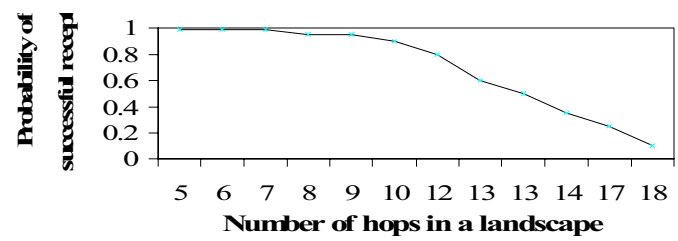

Figure 10: Probability of successful reception with the number of hops

Figure 10 shows the probability of successful reception with the number of hops. The number of hops is an "issue" metric since it is a "path metric" to enable reliability. Figure 10 shows that for high values in the

Each session consists of one or more downloads. This evaluation is taken for unfinished downloads in a session. 
number of hops, the end users can not experience successful reception of packets. In figure11 the percentage of successful reception is illustrated. The percentage of successful reception is shown compared with the number of alternations of cPIs during an active transmission. The percentage of successful reception does not drop as expected with the dynamic nature of moving users. It is shown that for almost 10 alternations the percentage of successful reception is above $68 \%$ which means that in any landscape one of two users could become PI, offering nearly $2 / 3$ of successful packet reception.

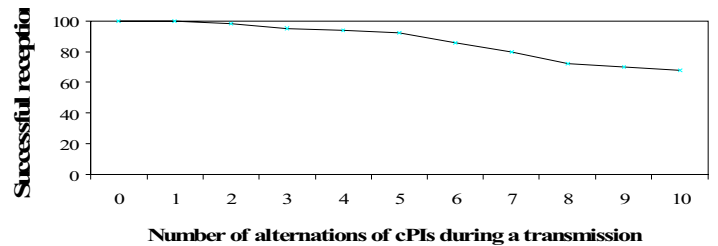

Figure 11: Percentage of successful reception with the number of alternations of cPIs during an active transmission.

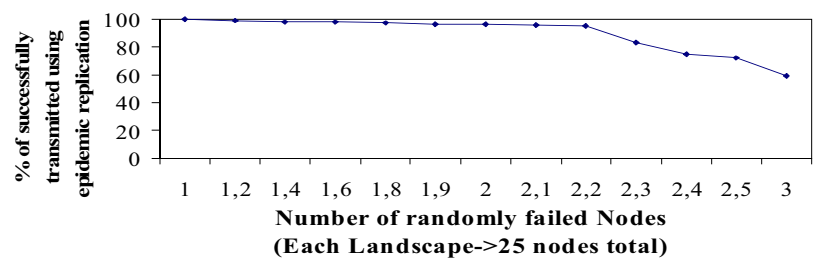

Figure 12: Mean number of randomly failed Nodes (in which a requested packet replica exists) with the number of successfully transmitted using epidemic data replication.

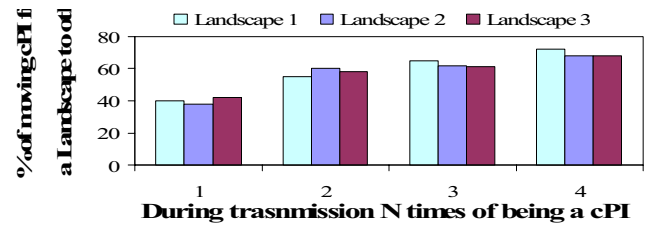

Figure 13: Percentage of moving cPI from a Landscape to other, with the number of times a user being a cPI during transmissions.

To evaluate thoroughly the exact performance we have examined the whole scenario under sudden node failures. As in real time wireless networks, device's failures could occur in a relatively small percentage of devices with respect to the total number of communicating nodes. In real time, wireless network's sudden failures supervene due to battery limitations (battery bugs-unpredictable shut down of device) and could lead to network partition (network split). However this scenario is expected that would substantially reduce the percentage of successfully transmitted files. In figure 12 it is easily recognizable that epidemic recursive data replication prevents sudden failures of nodes, preventing network partitioning and packet sharing failure. However if the number of failed nodes are more than 3 , the percentage of successfully transmitted is reduced progressively with the number of failed nodes which contain a requested replica.

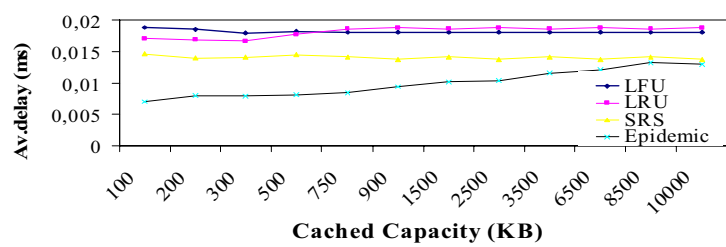

Figure 14: Average packet delay for different cache placement strategies and the corresponding cached capacity.

Figure13 illustrates the percentage of a moving cPI from a landscape to another, versus the number of times that a user could become a cPI during transmissions. This measure is extracted just for statistical reasons to evaluate each cPI and its possibility to move to nearby landscapes (directions for future research could evaluate and correlate the path of each device with "matching" criteria). When a transmission occurs each node statistically "passes" from cPI stage at least once every three independent transmissions. This means that each node might have high remaining energy and capacity (matching the criteria) and additionally almost $1 / 6$ of the nodes in a landscape changes landscape during that transmission. As an outcome to this, it would be expected that the packet loss measure will be increased. On the contrary the packet loss under the HyMIS scheme remains at satisfying levels (as seen in figure 5).

The average packet delay with the corresponding cached capacity for different cache placement strategies is shown in figure14. For the comparison we used the generic cache replacement policies of Least Recently Used (LRU), Size Replacement Strategy (SRS), Least Frequently Used (LFU). LRU is tested for when the copied file is least recently used (the cached item is associated with a time stamp). SRS is tested by aggregating the large files which in our scenario the concept large is equal to $0.33 *$ Total_remaining_capacity. Finally LFU strategy evicts the aggregation to cached files which are accessed least frequently. Figure 14 depicts that epidemic approach using the HyMIS structure behaves sensibly better for small files and satisfactory for relatively large files. Compared with the other implemented methods the epidemic approach with HyMIS structure responds significantly better in terms of average delay which is crucial metric for end-to-end QoS.

\section{Conclusions and further research}

In this work a reliable file sharing scheme is explored using the HyMIS structure. The proposed scheme is applied to a MP2P network without preinstalled and infrastructure components. HyMIS scheme proposes a novel approach for efficient 'interlandcast' resource 
sharing. Pure mobile Infostation concept is adopted for any node's implication process for being carrier of data packets to other nodes. The HyMIS architecture could be the basis for reliable file sharing among hybrid platforms in today's pervasive environments.

Future directions will be focused in MP2P network's connection with internet using the HyMIS and the static (modified) Infostations. An interesting open research issue is the severe examination of the anti-entropy nodes, or a history based framework for each device in a region and the estimated correlation in the epidemic dissemination process. Under certain circumstances and node characteristics there may be locality to the movement patterns of mobile nodes, as well as a correlation function with node's contribution in the diffusion process.

\section{References}

[1] D. J. Goodman, J. Borras, N.B. Mandayam, and R.D. Yates, "INFOSTATIONS: A New System for Data and Messaging Services," Proceedings of IEEE VTC '97 2 (1997) pp.969-973.

[2] S.-Y. Ni, Y.-C. Tseng, Y.-S. Chen, and J.-P. Sheu, "The broadcast storm problem in a mobile ad hoc network". In Proceedings of the Fifth Annual ACM/IEEE International Conference on Mobile Computing and Networking, pages 151-162. ACM Press, 1999.

[3] D. Ganesan, B. Krishnamachari, A. Woo, D. Culler, D. Estrin, and S. Wicker, "Complex behavior at scale: An experimental study of low-power wireless sensor networks". Technical Report UCLA/CSD-TR 02-0013, UCLA, 2002.

[4] A. Demers, D. Greene, C. Hauser, W. Irish, and J. Larson, "Epidemic algorithms for replicated database maintenance". In Proceedings of the Sixth Annual ACM Symposium on Principles of Distributed Computing, pages 1-12. ACM Press, 1987.

[5] J. Kulik, W. R. Heinzelman, and H. Balakrishnan, "Negotiationbased protocols for disseminating information in wireless sensor networks". Wireless Networks, 8(2-3):169-185, 2002.

[6] X. Hong, K. Xu, and M. Gerla, "Scalable routing protocols for mobile ad hoc networks". IEEE Network Magazine, 16(4):11 -21, Jul-Aug 2002.

[7] T. Hara, "Effective Replica Allocation in Ad Hoc Networks for Improving Data Accessibility," Proceedings of the IEEE INFOCOM 2001 Conference, pp. 1568-1576, 2001.

[8] J. Tchakarov and N. Vaidya, "Efficient content location in wireless ad hoc networks". IEEE International Conference on Mobile Data Management (MDM), 2004.

[9] C. D. Gavrilovich, G. Ware, L. Freindenrich, "Broadband Communication of the Higways of Tomorrow", IEEE Communications Magazine, April 2001.

[10] M. Grossglauser and D. Tse, "Mobility Increases the Capacity of Ad Hoc Wireless Networks", Proceedings of IEEE Infocom 2001, pp 312-319.

[11] A. Iacono and C. Rose, "Infostations: New Perspectives on Wireless Data Networks," WINLAB technical document, Rutgers University, 2000.

[12] O. Dousse, P. Thiran, and M. Hasler, "Connectivity in adhoc and hybrid networks," Proceedings of IEEE Infocom 2002, New York, 2002.

[13] T. Small and Z.J. Haas, "The Shared Wireless Infostation Model - A New Ad Hoc Networking Paradigm (or Where there is a
Whale, there is a Way)".Proceedings of the ACM MobiHoc 2003 conference, Annapolis, Maryland, 2003, pp 233-244.

[14] M. E. J. Newman, "The Movements of North Pacific Blue Whales During the Feeding Season off Southern California and their Southern Fall Migration" Santa Fe Institute 01-12-073.

[15] F. Brauer and C. Ch'avez, "Mathematical Models in Population Biology and Epidemiology" Springer-Verlag New York, Inc., 2001.

[16] T. Camp, J. Boleng, and V. Davies, "A survey of mobility models for ad hoc network research". Wireless Communications \& Mobile Computing (WCMC): Special issue on Mobile Ad Hoc Networking: Research, Trends and Applications, 2(5):483-502, 2002.

[17] K. Park, G. Kim, and M. Crovella, "On the relationship between file sizes, transport protocols and self-similar network traffic". Proceedings of ICNP 1996,pp 171-179.

[18] P. Ulanovs, E. Petersons. "Modeling methods of self-similar traffic for network performance evaluation", Scientific Proceedings of RTU. Series 7. Telecommunications and Electronics, 2002.

[19] Z. Haas and M. Pearlman, "The performance of query control schemes for the zone routing protocol". ACM/IEEE Transactions on Networking 9(4), 2001, pp. 427-438.

[20] Z. Haas and M. Pearlman. "The Zone Routing Protocol (ZRP) for Ad Hoc Networks", Internet Draft, draft-ietf-manet-zone-zrp02.txt, June 1999.

[21] C. Mavromoustakis, H. Karatza, "Adaptive Traffic-based Control Method for Energy Conservation in Wireless Devices". Journal of Simulation Practice and Theory (SIMPRA), Elsevier, Volume 13, Issue 3, April 2005, pp 213-232.

[22] Constandinos X. Mavromoustakis and Helen D. Karatza, "Segmented File Sharing with Recursive Epidemic Placement Policy for Reliability in Mobile Peer-to-Peer Devices". Proceedings of the 13th Annual Meeting of the IEEE International Symposium on Modeling, Analysis, and Simulation of Computer and Telecommunication Systems (MASCOTS), Georgia Tech, Atlanta, Georgia, September 26-29, 2005, pp 371-380. 